\title{
Representing quantum structures as near semirings
}

\author{
STEFANO BONZIO*, University of Cagliari, Italy.
}

IVAN CHAJDA**, Palacký University, Olomouc, Czech Republic.

ANTONIO LEDDA ${ }^{\dagger}$, University of Cagliari, Italy.

\begin{abstract}
In this article, we introduce the notion of near semiring with involution. Generalizing the theory of semirings we aim at represent quantum structures, such as basic algebras and orthomodular lattices, in terms of near semirings with involution. In particular, after discussing several properties of near semirings, we introduce the so-called Lukasiewicz near semirings, as a particular case of near semirings, and we show that every basic algebra is representable as (precisely, it is term equivalent to) a near semiring. In the particular case in which a Lukasiewicz near semiring is also a semiring, we obtain as a corollary a representation of MV-algebras as semirings. Analogously, by introducing a particular subclass of Łukasiewicz near semirings, that we termed orthomodular near semirings, we obtain a representation of orthomodular lattices. In the second part of the article, we discuss several universal algebraic properties of Łukasiewicz near semirings and we show that the variety of involutive integral near semirings is a Church variety. This yields a neat equational characterization of central elements of this variety. As a byproduct of such, we obtain several direct decomposition theorems for this class of algebras.
\end{abstract}

Keywords: Near semiring, involution, Łukasiewicz near semiring, semiring, basic algebra, orthomodular lattice, MV-algebra, Church variety, central element.

\section{Introduction}

It is a long-dated result, due to Marshall Stone [19], that the theory of Boolean algebras (the algebraic counterpart of classical logic) can be framed within the theory of rigs, through the concept of Boolean ring. More recently, in the last decade, the relations between prominent algebraic structures from many-valued logics and (semi)ring theory have stirred a renewed attention (see, e.g. [1, 13]). It was shown by Belluce, Di Nola, Ferraioli [2] and Gerla [13] that MV-algebras (the algebraic semantics of infinite-valued Łukasiewicz logic) can be viewed as particular semirings: MV-semirings. The results achieved in the MV-algebras' context are extremely interesting and promising. Quoting A. Di Nola and C. Russo [12]:

[...] besides serving MV-algebra theory they suggest a possible payback, namely, that MValgebras can on their turn give ideas and tools to semiring and semifield theories. It is worth noticing also that, as well as MV-algebras, various other logic-related algebraic structures can be viewed as special idempotent semirings, and therefore this approach could be further extended.

\footnotetext{
*E-mail: stefano.bonzio@gmail.com

**E-mail: ivan.chajda@upol.cz

†E-mail: antonio.ledda@unica.it
} 


\section{Near semirings}

Taking up their suggestion, we will show in this article that this method can be fruitfully raised to a considerably general level. Indeed, we will see that a number of algebraic structures of major importance to non-classical logics are representable as semiring-like structures.

This article will be mainly focused on basic algebras and orthomodular lattices. Basic algebras were introduced by R. Halaš, J. Kühr and one of the authors of this article as a common generalization of both MV-algebras - the algebraic alter-ego of Łukasiewicz many-valued logic - and orthomodular lattices (the interested reader may consult [5] and [7] for details) - the algebraic counterpart of the logic of quantum mechanics (for an extensive discussion we refer to [3, 15]). Inspired by the results in [2], it seems natural to hunt for an appropriate notion that would play, in the wider domain of Basic algebras, the same role that MV-semirings interpret in the context of MV-algebras. We believe that this concept will shine some light on the theory both of MV-algebras and orthomodular lattices. It will be interesting, in fact, to examine how this general notion specifies to context so far apart from each other. Indeed, MV-algebras and orthomodular lattices. We will see that this task is far from straightforward. Indeed, Basic algebras can not be represented as semirings since they do not satisfy both distributivity laws, but right-distributivity only; in addition, multiplication need not to be associative in general.

These observations seem to suggest that a substantial weakening of the concept of semiring would be required to embrace such algebras. An appropriate generalization can be found in $[9,10]$ where $\mathrm{H}$. Länger and one of the present authors discuss the concept of near semiring. Taking up ideas from [2] and [11], in order to provide a semiring-like representation of basic algebras, we specialize the concept of near semiring and introduce the notion of Łukasiewicz near semiring and orthomodular near semiring.

The article is structured as follows: in Section 2 we introduce the notions of near semiring, near semiring with involution and Łukasiewicz near semiring and discuss some basic properties of these three classes. In Section 3 after a concise presentation of basic algebras, we prove that they can be represented by Łukasiewicz near semirings. In Section 4 we discuss several universal algebraic properties of Łukasiewicz near semirings: congruence regularity, congruence permutability and congruence distributivity. In Section 5 we introduce the concept of orthomodular near semiring, and we show that orthomodular lattices can be represented by of these algebraic structures. Finally, in Section 6, we claim that the variety of involutive integral near semirings is a Church variety [18]. This yields an explicit description of central elements and, consequently, a series of direct decomposition theorems.

\section{Near semirings}

DeFinition 1

A near semiring is an algebra $\mathbf{R}=\langle R,+, \cdot, 0,1\rangle$ of type $\langle 2,2,0,0\rangle$ such that

(i) $\langle R,+, 0\rangle$ is a commutative monoid;

(ii) $\langle R, \cdot, 1\rangle$ is a groupoid satisfying $x \cdot 1=x=1 \cdot x$ (a unital groupoid);

(iii) $(x+y) \cdot z=(x \cdot z)+(y \cdot z)$;

(iv) $x \cdot 0=0 \cdot x=0$.

We will refer to the operations + and - as sum and multiplication, respectively, and we call the identity in (iii) right distributivity. Near semirings generalize semirings to a non-associative and weaklydistributive context. Indeed, a semiring is a near semiring such that $\langle R, \cdot, 1\rangle$ is a monoid (i.e. · is also associative) that satisfies left distributivity: $x \cdot(y+z)=(x \cdot y)+(x \cdot z)$, for all $x, y, z \in R$. Throughout 


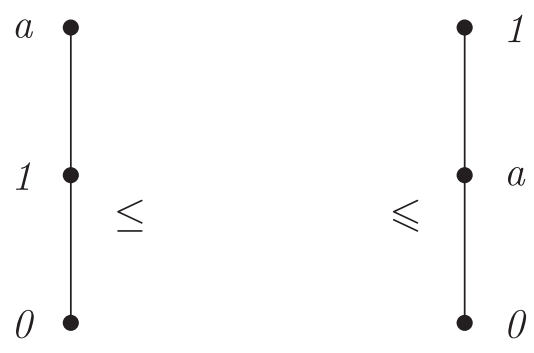

FIG. 1. The Hasse diagrams of the partial orders induced by sum, $\leq$ (left-hand side), and multiplication, $\leqslant$ (right-hand side).

the article, a near semiring $\mathbf{R}$ is called associative if it satisfies $(x \cdot y) \cdot z=x \cdot(y \cdot z)$, commutative if it satisfies $x \cdot y=y \cdot x$; idempotent if it satisfies $x+x=x$ and integral if $x+1=1$ holds.

REMARK 1

Let $\mathbf{R}$ be an idempotent near semiring. Then $\langle R,+\rangle$ is a semilattice. In particular, $\langle R,+\rangle$ can be considered as a join-semilattice, where the induced order is defined as $x \leq y$ iff $x+y=y$ and the constant 0 is the least element. Moreover, whenever $\mathbf{R}$ is integral, the constant 1 is the greatest element with respect to the induced order $\leq$.

REMARK 2

Let $\mathbf{R}$ be an idempotent commutative semiring, whose multiplication is also idempotent $(x \cdot x=x)$. Then, $\langle R, \cdot\rangle$ is also a semilattice, in particular a meet-semilattice. Notice that, in general, $\langle R,+, \cdot\rangle$ need not be a lattice. Indeed, the absorption laws may fail. ${ }^{1}$ Moreover, the order induced by the multiplication, $x \leqslant y$ iff $x \cdot y=x$, may differ from $\leq$, as Example 1 shows.

EXAMPLE 1

Let $\mathbf{R}$ be the near semiring whose universe is $R=\{0,1, a\}$ and whose sum and multiplication are defined in the following tables:

\begin{tabular}{c|ccc}
+ & 0 & $a$ & 1 \\
\hline 0 & 0 & $a$ & 1 \\
$a$ & $a$ & $a$ & $a$ \\
1 & 1 & $a$ & 1
\end{tabular}

\begin{tabular}{c|ccc}
$\cdot$ & 0 & $\mathrm{a}$ & 1 \\
\hline 0 & 0 & 0 & 0 \\
$a$ & 0 & $a$ & $a$ \\
1 & 0 & $a$ & 1
\end{tabular}

It is not difficult to verify that $\mathbf{R}$ is both additively and multiplicatively idempotent, commutative and associative; therefore, $\langle R, \cdot\rangle$ is a meet-semilattice. However, $\mathbf{R}$ is not integral, since $a+1=a \neq 1$; and the absorption laws do not hold: $1 \cdot(a+1)=1 \cdot a=a \neq 1$. It can be seen in Figure 1 that the orders induced by + and $\cdot$ are different.

The following lemma states that in any near semiring, multiplication is monotone on the right-hand side, due to right distributivity.

\section{LEMMA 1}

Let $\mathbf{R}$ be a near semiring. Then, $x \leq y$ implies $x \cdot z \leq y \cdot z$.

Proof. Suppose $x \leq y$, i.e. $x+y=y$. Therefore $y \cdot z=(x+y) \cdot z=(x \cdot z)+(y \cdot z)$, which implies that $x \cdot z \leq y \cdot z$.

\footnotetext{
${ }^{1}$ Let us remark that, since near semirings satisfy right distributivity only, we may have different forms of absorption.
} 


\section{Near semirings}

Since near semirings are, in general, not distributive (left distributivity does not hold), multiplication is not monotone in the left component. However, there will be cases in which distributivity holds for some special elements.

DEFINITION 2

Let $\langle R,+, \cdot, 0,1\rangle$ be an idempotent near semiring, with $\leq$ the induced order. A map $\alpha: R \rightarrow R$ is called an involution on $\mathrm{R}$ if it satisfies the following conditions for each $x, y \in R$ :

(a) $\alpha(\alpha(x))=x$;

(b) if $x \leq y$ then $\alpha(y) \leq \alpha(x)$.

The algebra $\mathbf{R}=\langle R,+, \cdot, 0,1, \alpha\rangle$ will be called an involutive near semiring.

Sometimes, if no confusion is possible, we will write $\alpha \alpha x$ in place of $\alpha(\alpha(x))$. Some basic arithmetical properties of involutive near semirings are presented in the following lemma.

LEMMA 2

Let $\mathbf{R}$ be an involutive near semiring. Then

(i) $\alpha(x+y)+\alpha(x)=\alpha(x)$.

(ii) $\mathbf{R}$ is integral if and only if $\alpha(0)=1$ (and consequently $\alpha(1)=0$ ).

Proof. (i) Since + is idempotent, we have that $\alpha(x)=\alpha(x)+\alpha(x)$. Moreover, since $x+y=(x+x)+$ $y=x+(x+y), x \leq x+y$ and $\alpha$ is an involution on $R, \alpha(x+y) \leq \alpha(x)$. Therefore $\alpha(x+y)+\alpha(x) \leq$ $\alpha(x)+\alpha(x)=\alpha(x)$. The converse $\alpha(x) \leq \alpha(x)+\alpha(x+y)$ holds because $\langle R,+\rangle$ is a join-semilattice as we noticed in Remark 1.

(ii) Suppose that $\mathbf{R}$ is integral, i.e. $x \leq 1$ for each $x \in R$, then $\alpha(1) \leq \alpha(x)$. Since $\alpha$ is an involution we have that $\alpha(1)+x=x$ for each $x \in R$, which means that $\alpha(1)$ is a neutral element with respect to the sum and since $\langle R,+, 0\rangle$ is a (commutative) monoid, the neutral is unique ${ }^{2}$, thus $\alpha(1)=0$ and $\alpha(0)=1$. For the converse, suppose $\alpha(0)=1$. Then, by (i), we have that $\alpha(x+y) \leq \alpha(x)$, which, for $x=0$, implies $\alpha(y) \leq \alpha(0)=1$, which means that for each $x \in R$ we have $x \leq 1$, i.e. $\mathbf{R}$ is integral.

\section{REMARK 3}

Notice that, in general, $x \leq \alpha(0)$. However, it is not true that in any involutive near semiring $\alpha(0)=1$ (see Example 2). In fact, there are cases of non integral involutive near semiring

EXAMPLE 2

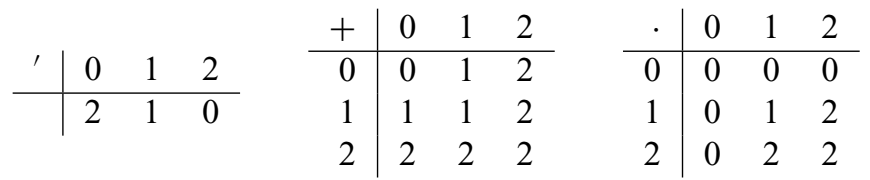

THEOREM 1

Let $\mathbf{R}$ be an involutive near semiring and define two new operations as $x+{ }_{\alpha} y=\alpha(\alpha(x)+\alpha(y))$ and $x \cdot{ }_{\alpha} y=\alpha(\alpha(x) \cdot \alpha(y))$. Then:

(a) $x+y=\alpha\left(\alpha(x)+{ }_{\alpha} \alpha(y)\right), x \cdot y=\alpha(\alpha(x) \cdot \alpha \alpha(y))$;

(b) $\mathbf{R}_{\alpha}=\left\langle R,+_{\alpha},{ }_{\alpha}, \alpha, \alpha(0), \alpha(1)\right\rangle$ is an involutive near semiring.

Proof. (a) By definition of $+_{\alpha}$ we have that $\alpha\left(\alpha(x)+{ }_{\alpha} \alpha(y)\right)=\alpha \alpha(\alpha \alpha(x)+\alpha \alpha(y))=x+y$. The proof runs analogously for $\cdot \alpha$.

\footnotetext{
${ }^{2}$ This is in fact true for semigroups.
} 
(b) We start by showing that $\left\langle R,+_{\alpha}, \alpha(0)\right\rangle$ is a commutative monoid. Commutativity of $+_{\alpha}$ trivially follows by definition. Furthermore:

$$
\begin{array}{ll}
\left(x+{ }_{\alpha} y\right)+{ }_{\alpha} z=\alpha\left(\alpha\left(x+{ }_{\alpha} y\right)+\alpha(z)\right) & \left(\text { Def. }+_{\alpha}\right) \\
=\alpha(\alpha \alpha(\alpha(x)+\alpha(y))+\alpha(z)) & \left(\text { Def. }+_{\alpha}\right) \\
=\alpha((\alpha(x)+\alpha(y))+\alpha(z)) & \text { (Inv.) } \\
=\alpha(\alpha(x)+(\alpha(y)+\alpha(z))) & \left(\text { Def. }+{ }_{\alpha}\right) \\
=\alpha\left(\alpha(x)+\alpha\left(y+{ }_{\alpha} z\right)\right) & \left(\text { Def }+_{\alpha}\right) \\
=x+{ }_{\alpha}\left(y+{ }_{\alpha} z\right) &
\end{array}
$$

proving associativity of $+_{\alpha}$. Finally,

$$
\begin{array}{ll}
x+{ }_{\alpha} \alpha(0)=\alpha(\alpha(x)+\alpha(\alpha(0))) & \left(\text { Def. }+_{\alpha}\right) \\
=\alpha(\alpha(x)+0) & \text { (Inv.) } \\
=\alpha(\alpha(x))=x & \text { (Monoid) }
\end{array}
$$

The fact that $\alpha(0)$ is also a left neutral follows from commutativity. The proof of the fact that $\langle R, \cdot \alpha, \alpha(1)\rangle$ is a groupoid with $\alpha(1)$ as neutral element is analogous.

Furthermore, $\quad x \cdot \alpha \alpha(0)=\alpha(\alpha(x) \cdot \alpha(\alpha(0))=\alpha(\alpha(x) \cdot 0)=\alpha(0)$ and similarly to show that $\alpha(0) \cdot{ }_{\alpha} x=\alpha(0)$.

It only remains to show that right distributivity holds.

$$
\begin{aligned}
& \left(x+{ }_{\alpha} y\right) \cdot{ }_{\alpha} z=\alpha\left(\alpha\left(x+{ }_{\alpha} y\right) \cdot \alpha(z)\right) \\
& =\alpha(\alpha \alpha(\alpha(x)+\alpha(y)) \cdot \alpha(z)) \\
& =\alpha((\alpha(x)+\alpha(y)) \cdot \alpha(z)) \\
& =\alpha((\alpha(x) \cdot \alpha(z))+(\alpha(y) \cdot \alpha(z))) \\
& =\left(x \cdot \cdot_{\alpha} z\right)+_{\alpha}\left(y \cdot{ }_{\alpha} z\right)
\end{aligned}
$$

Therefore $\left\langle R,+_{\alpha},{ }_{\alpha}, \alpha(0), \alpha(1)\right\rangle$ is a near semiring.

In general, for a given near semiring $\mathbf{R}$, we will refer to $\mathbf{R}_{\alpha}$ as the dual near semiring.

As we mentioned in the introduction, semiring-like structures are relevant to the theory of prominent algebraic structures from many-valued logics. In particular, it was shown by Belluce, Di Nola, Ferraioli [2] and Gerla [13] that MV-algebras can be represented as semirings. Following the same idea, we aim at representing some algebraic structures deriving from quantum logics as near semirings. For this reason we introduce the notion of Łukasiewicz near semiring.

DEFINITION 3

Let $\mathbf{R}$ be an involutive near semiring. $\mathbf{R}$ is called a Lukasiewicz near semiring if it satisfies the following additional identity

(Ł) $\alpha(x \cdot \alpha(y)) \cdot \alpha(y)=\alpha(y \cdot \alpha(x)) \cdot \alpha(x)$.

A semiring satisfying ( $($ ) will be called a Lukasiewicz semiring.

Identity (Ł), in Definition 3, clearly reflects Łukasiewicz identity in the standard axiomatization of MV-algebras. As already noticed, the constant 1 need not necessarily be the top element with respect to the order $\leq$ in general. However, as the next lemma shows, this is always the case for Łukasiewicz near semiring. This fact will be frequently used throughout this article. 
6 Near semirings

LEMMA 3

Let $\mathbf{R}$ be Łukasiewicz near semiring. Then

(a) $x \cdot \alpha(x)=\alpha(x) \cdot x=0$;

(b) $\mathbf{R}$ is integral;

(c) $x \cdot \alpha(x+y)=0$;

(d) $(x+y) \cdot \alpha(x)=y \cdot \alpha(x)$;

(e) $x+y=\alpha(\alpha(x \cdot \alpha(y)) \cdot \alpha(y))$.

Proof. (a) Let us observe that, upon setting $x=0$ and $y=1$ in ( $\mathrm{E})$, we get $\alpha(0) \cdot \alpha(1)=\alpha(0 \cdot \alpha(1))$. $\alpha(1)=\alpha(1 \cdot \alpha(0)) \cdot \alpha(0)=\alpha(\alpha(0)) \cdot \alpha(0)=0$. Since 0 is the unit with respect to the sum, we have that $0+\alpha(x)=\alpha(x)$, i.e. $0 \leq \alpha(x)$. Therefore $x \leq \alpha(0)$ and then $x+\alpha(0)=\alpha(0)$. Using these two facts, we obtain

$$
\begin{aligned}
& 0=(x+\alpha(0)) \cdot \alpha(1) \\
& =(x \cdot \alpha(1))+(\alpha(0) \cdot \alpha(1)) \\
& =(x \cdot \alpha(1))+0 \\
& =x \cdot \alpha(1)
\end{aligned}
$$

We finally get

$$
\begin{aligned}
& x \cdot \alpha(x)=\alpha(\alpha(x)) \cdot \alpha(x) \\
& =\alpha(1 \cdot \alpha(x)) \cdot \alpha(x) \\
& =\alpha(x \cdot \alpha(1)) \cdot \alpha(1) \\
& =\alpha(0) \cdot \alpha(1) \\
& =0 .
\end{aligned}
$$

(Distr.)

Since $\alpha$ is an involution, it follows that also $\alpha(x) \cdot x=0$.

(b) $\alpha(1)=1 \cdot \alpha(1)=0$, by (a). Then by Lemma 2 (ii) we have that $\mathbf{R}$ is integral.

(c) Since $x \leq x+y$. Then, by Lemma $1, x \cdot \alpha(x+y) \leq(x+y) \cdot \alpha(x+y)=0$.

(d) It is enough using right distributivity and (a), indeed $(x+y) \cdot \alpha(x)=(x \cdot \alpha(x))+(y \cdot \alpha(x))=$ $0+(y \cdot \alpha(x))=y \cdot \alpha(x)$.

(e)

$$
\begin{aligned}
& \alpha(x \cdot \alpha(y)) \cdot \alpha(y)=\alpha(y \cdot \alpha(x)) \cdot \alpha(x) \\
& =\alpha((x+y) \cdot \alpha(x)) \cdot \alpha(x) \\
& =\alpha(x \cdot \alpha(x+y)) \cdot \alpha(x+y) \\
& =\alpha(0) \cdot \alpha(x+y) \\
& =1 \cdot \alpha(x+y) \\
& =\alpha(x+y) .
\end{aligned}
$$

Therefore $x+y=\alpha(\alpha(x \cdot \alpha(y)) \cdot \alpha(y))$

The next lemma shows that, in the specific case of Łukasiewicz near semirings, the order induced by the sum is equivalently expressed by multiplication. 
LEMMA 4

Let $\mathbf{R}$ be a Łukasiewicz near semiring. Then $x \leq y$ if and only if $x \cdot \alpha(y)=0$.

Proof. Let $a \leq b$, for some $a, b \in R$. Then $a+b=b$ and, by Lemma 3-(c), we get that $0=a \cdot \alpha(a+b)=$ $a \cdot \alpha(b)$.

Conversely, suppose that $a \cdot \alpha(b)=0$ for some $a, b \in R$.

$$
\begin{array}{ll}
a+b=\alpha(\alpha(a \cdot \alpha(b)) \cdot \alpha(b))) & \text { (Lemma 3(e)) } \\
=\alpha(\alpha(0) \cdot \alpha(b)) & \text { (Assumption) } \\
=b . &
\end{array}
$$

Therefore $a \leq b$.

Theorem 2 clarifies the connection between Łukasiewicz near semirings and Łukasiewicz semirings.

\section{THeOREM 2}

Let $\mathbf{R}$ be a Łukasiewicz near semiring whose multiplication is associative. Then multiplication is also commutative, and therefore $\mathbf{R}$ is a commutative Łukasiewicz semiring.

Proof. Suppose $\langle R, \cdot\rangle$ is a semigroup. Then

$$
\begin{aligned}
& \alpha(x \cdot y) \cdot(y \cdot x)=(\alpha(x \cdot y) \cdot y) \cdot x \\
& =(\alpha(\alpha(y) \cdot \alpha(x)) \cdot \alpha(x)) \cdot x \\
& =(\alpha(\alpha(y) \cdot \alpha(x)) \cdot(\alpha(x) \cdot x) \\
& =(\alpha(\alpha(y) \cdot \alpha(x)) \cdot 0 \\
& =0 .
\end{aligned}
$$

Therefore $\alpha(x \cdot y) \cdot(y \cdot x)=0$. Analogously, $\alpha(y \cdot x) \cdot(x \cdot y)=0$. Applying Lemma 4 to both the equations, we obtain that $\alpha(x \cdot y) \leq \alpha(y \cdot x)$ and $\alpha(y \cdot x) \leq \alpha(x \cdot y)$. Therefore $\alpha(x \cdot y)=\alpha(y \cdot x)$, i.e. $x \cdot y=y \cdot x$. Therefore, multiplication commutes. Hence, to prove that $\mathbf{R}$ is a Łukasiewicz semiring, it suffices to observe that left distributivity follows straight away from right distributivity.

As an immediate consequences of the previous result we obtain that

COROLlary 1

Every Łukasiewicz semiring is commutative.

\section{Corollary 2}

A Łukasiewicz near semiring is a Łukasiewicz semiring if and only if multiplication is associative.

By Lemma 3, any Łukasiewicz near semiring is integral. Therefore, it makes sense to introduce the notion of interval on a Lukasiewicz near semiring $\mathbf{R}$ : $[a, 1]=\{x \in R \mid a \leq x\}$. The next result shows that any such interval can be equipped with an antitone involution.

\section{THEOREM 3}

Let $\mathbf{R}$ be a Łukasiewicz near semiring, $\leq$ the induced order, and $a \in R$. The map $h_{a}:[a, 1] \rightarrow[a, 1]$, defined by $x \mapsto x^{a}=\alpha(x \cdot \alpha(a))$ is an antitone involution on the interval $[a, 1]$.

Proof. We first show that $h_{a}$ is well defined. Indeed, since $\mathbf{R}$ is integral (Lemma 3 ) we have that $x \leq 1$, thus $x \cdot \alpha(a) \leq 1 \cdot \alpha(a)$ by monotonicity, then $a=\alpha(\alpha(a))=\alpha(1 \cdot \alpha(a)) \leq \alpha(x \cdot \alpha(a))=x^{a}$, i.e. $x^{a} \in[a, 1]$. 


\section{Near semirings}

Moreover, $h_{a}$ is antitone. Suppose $x, y \in[a, 1]$ with $x \leq y$. Since multiplication is monotone (Lemma 1) we get that $x \cdot \alpha(a) \leq y \cdot \alpha(a)$. Therefore $y^{a}=\alpha(y \cdot \alpha(a)) \leq \alpha(x \cdot \alpha(a))=x^{a}$, i.e. $h_{a}$ is antitone.

Since for any $x \in[a, 1], a \leq x$, i.e. $a+x=x$, then by Lemma 3-(c)

$$
a \cdot \alpha(x)=a \cdot \alpha(a+x)=0
$$

From this fact we obtain that:

$$
\begin{aligned}
& x^{a a}=\alpha\left(x^{a} \cdot \alpha(a)\right)=\alpha(\alpha(x \cdot \alpha(a)) \cdot \alpha(a)) \\
& =\alpha(\alpha(a \cdot \alpha(x)) \cdot \alpha(x)) \\
& =\alpha(\alpha(0) \cdot \alpha(x)) \\
& =\alpha(1 \cdot \alpha(x)) \\
& =\alpha(\alpha(x))=x .
\end{aligned}
$$

This shows that $h_{a}$ is an antitone involution on the interval $[a, 1]$.

Let us observe that, in $[5,8]$, the involution constructed in Theorem 3 is termed sectional involution.

\section{Basic algebras as near semirings}

Basic algebras, introduced in the last decade by Halaš, Kühr, and one of the authors of this article, as a common generalization of both MV-algebras and orthomodular lattices. They can be regarded as a non-associative and non-commutative generalization of MV algebras. These algebras are in bijective correspondence with bounded lattices having an antitone involution on every principal filter (sectional antitone involutions). An introductory as well as comprehensive survey on basic algebras can be found in [5].

In this section, we discuss the relations between Łukasiewicz near semirings and basic algebras. Let us recall that a basic algebra is an algebra $\mathbf{A}=\left\langle A, \oplus,{ }^{\prime}, 0\right\rangle$ satisfying the following identities:

(BA1) $x \oplus 0=x$;

(BA2) $x^{\prime \prime}=x$

(BA3) $\left(x^{\prime} \oplus y\right)^{\prime} \oplus y=\left(y \oplus x^{\prime}\right)^{\prime} \oplus x$;

(BA4) $\left(\left((x \oplus y)^{\prime} \oplus y\right)^{\prime} \oplus z\right)^{\prime} \oplus(x \oplus z)=1$.

where $0^{\prime}=1$ and (BA3) is the Eukasiewicz identity. It is not difficult to show that every MV algebra is a basic algebra. More precisely, the class of MV algebras is a subvariety of the variety of basic algebras and it is axiomatized by the identity expressing associativity of $\oplus$ (for details see [5]). ${ }^{3}$ Every basic algebra is in fact a bounded lattice, where the lattice order is defined by $x \leq y$ iff $x^{\prime} \oplus y=1$, the join operation is defined by $x \vee y=\left(x^{\prime} \oplus y\right)^{\prime} \oplus y$, while the meet is defined á la de Morgan: $x \wedge y=\left(x^{\prime} \vee y^{\prime}\right)^{\prime}$. It can be verified that 0 and 1 are the bottom and the top elements, respectively, of the lattice.

Conversely, let us remark that, in any bounded lattice with sectional antitone involutions $\left\langle L, \vee, \wedge,\left({ }^{a}\right)_{a \in L}, 0,1\right\rangle$ (the interested reader may consult [7], [5] ), it is possible to define the operations

$$
x^{\prime}=x^{0}, x \oplus y:=\left(x^{0} \vee y\right)^{y},
$$

such that $\left\langle L, \oplus,,^{\prime}, 0,1\right\rangle$ is a basic algebra. It can be proven that this correspondence is one to one. We will use this fact to establish a correspondence between Łukasiewicz near semirings and basic

\footnotetext{
${ }^{3}$ Indeed, it is shown in [5] that if $\oplus$ is associative then it is also commutative.
} 
algebras. In particular, we will discuss how basic algebras can be represented in terms of near semirings.

\section{THEOREM 4}

If $\mathbf{R}$ is a Łukasiewicz near semiring, then the structure $\mathbf{B}(\mathbf{R})=\langle R, \oplus, \alpha, 0\rangle$, where $x \oplus y$ is defined by $\alpha((\alpha(x)+y) \cdot \alpha(y))$ is a basic algebra.

Proof. The reduct $\langle R,+, 1\rangle$ is a (join) semilattice whose top element is 1 (Remark 1 and Lemma 3(b)). From Theorem 1, we have that $\left\langle R,+_{\alpha}\right\rangle$ is the dual meet semilattice. Therefore $\left\langle R,+,+_{\alpha}, 0,1\right\rangle$ is a bounded lattice. Furthermore, by Theorem 3 the map $x \mapsto x^{a}=\alpha(x \cdot \alpha(a))$ is an antitone involution on the interval $[a, 1]$ for all $a \in R$. So $\left\langle R,+,+_{\alpha},\left({ }^{a}\right)_{a \in R}, 0,1\right\rangle$ is a bounded involution lattice with sectional antitone involutions. And therefore it can be made into a basic algebra upon setting the operations as in equations (3.1). It follows that $x^{0}=x^{\prime}=\alpha(x)$ and $x \oplus y=\alpha((\alpha(x)+y) \cdot \alpha(y))$.

The next result shows a converse of the previous theorem: any basic algebra induces a Łukasiewicz near semiring.

\section{THEOREM 5}

If $\mathbf{B}=\left\langle B, \oplus,{ }^{\prime}, 0\right\rangle$ is a basic algebra, then the structure $\mathbf{R}(\mathbf{B})=\langle B,+, \cdot, \alpha, 0,1\rangle$, where $x+y, x \cdot y$ and $\alpha(x)$ are defined by $\left(x^{\prime} \oplus y\right)^{\prime} \oplus y,\left(x^{\prime} \oplus y^{\prime}\right)^{\prime}, x^{\prime}$, and $1=0^{\prime}$, respectively, is a Łukasiewicz near semiring.

Proof. As we mentioned, in any basic algebras $\left(x^{\prime} \oplus y\right)^{\prime} \oplus y$ defines a join-semilattice, whose least and greatest elements are, respectively, 0 and 1 . This assures that $\langle R,+, 0\rangle$ is a commutative monoid. Furthermore, it was shown in [5] that $(x+y) \cdot z=(x \cdot z)+(y \cdot z)$. Let us now prove that 1 is a neutral element for multiplication. We note that

$$
\begin{aligned}
& x \cdot 1=\left(x^{\prime} \oplus 1^{\prime}\right)^{\prime} \\
& =\left(x^{\prime} \oplus 0\right)^{\prime} \\
& =x^{\prime \prime} \\
& =x
\end{aligned}
$$

Upon observing that, in basic algebras, $x \oplus 0=x$ (BA1) implies $0 \oplus x=x$, then one analogously proves that $1 \cdot x=x$. To prove that 0 is an annhilator of multiplication, we show that

$$
\begin{aligned}
& x \cdot 0=\left(x^{\prime} \oplus 0^{\prime}\right)^{\prime} \\
& =\left(x^{\prime} \oplus 1\right)^{\prime} \\
& =1^{\prime} \\
& =0 .
\end{aligned}
$$

The proof that $0 \cdot x=0$ is analogous. Therefore $\mathbf{R}(\mathbf{B})$ is a near semiring. Since $\alpha(x)=x^{\prime}$, it is clear that it is also an antitone involution. We are left with checking that $\mathbf{R}(\mathbf{B})$ satisfies the conditions of a Lukasiewicz near semiring, Definition 3.

As regards condition (Ł),

$$
\begin{aligned}
& \alpha(x \cdot \alpha(y)) \cdot \alpha(y)=\left((x \oplus y)^{\prime} \oplus y\right)^{\prime} \\
& =\left((y \oplus x)^{\prime} \oplus x\right)^{\prime} \\
& =\alpha(y \cdot \alpha(x)) \cdot \alpha(x)
\end{aligned}
$$

This concludes the proof that $\mathbf{R}(\mathbf{B})$ is a Łukasiewicz near semiring. 
The results above state a correspondence between near Łukasiewicz semirings and basic algebras. In order to analyse these maps properly, we will refer to the variety of basic algebras and of Łukasiewicz near semiring as $\mathcal{B}$ and $\mathcal{R}$, respectively. In Theorem 5 we considered a map $f: \mathcal{B} \rightarrow \mathcal{R}$ associating to each basic algebra a Łukasiewicz near semiring $\mathbf{R}(\mathbf{B})$. On the other hand, in Theorem 4, we applied a map $g: \mathcal{R} \rightarrow \mathcal{B}$, associating to any Łukasiewicz near semiring $\mathbf{R}$ a basic algebra $\mathbf{B}(\mathbf{R})$.

The next theorem shows that $\mathbf{B}(\mathbf{R}(\mathbf{B}))$ actually coincides with $\mathbf{B}$ and, viceversa, $\mathbf{R}$ coincides with $\mathbf{R}(\mathbf{B}(\mathbf{R}))$.

\section{THEOREM 6}

The maps $f$ and $g$ are mutally inverse.

Proof. We start by checking that $\mathbf{B}(\mathbf{R}(\mathbf{B}))=\mathbf{B}$. We first note that $f\left(x^{\prime}\right)=\alpha(x)$ and $g(\alpha(x))=x^{\prime}$. Therefore $f(g(\alpha(x)))=\alpha(x)$ and $g\left(f\left(x^{\prime}\right)\right)=x^{\prime}$. We have to prove that $x \oplus y=x \widehat{\oplus} y$, where by $x \widehat{\oplus} y$ we indicate the sum in $\mathbf{B}(\mathbf{R}(\mathbf{B}))$. We use the fact that $\mathbf{R}(\mathbf{B})$ is a Lukasiewicz near semiring (Theorem 5), whose sum and multiplication are indicated by $\widehat{+}$ and $\widehat{\cdot}$, respectively.

$$
\begin{array}{ll}
x \widehat{\oplus} y=\alpha((\alpha(x) \widehat{+} y) \widehat{\cdot} \alpha(y)) & \text { (Def.) } \\
=\alpha((\alpha(x) \widehat{\cdot} \alpha(y)) \widehat{+}(\widehat{y \cdot \alpha}(y))) & \text { (Distr.) } \\
=\alpha((\alpha(x) \widehat{\cdot} \alpha(y)) \widehat{+} 0) & \text { (Lemma 3) } \\
=\alpha((\alpha(x) \widehat{\cdot} \alpha(y))=x \oplus y . &
\end{array}
$$

This is enough to have that $\mathbf{B}(\mathbf{R}(\mathbf{B}))=\mathbf{B}$. To see that $\mathbf{R}(\mathbf{B}(\mathbf{R}))=\mathbf{R}$ we need to check that $x \widehat{+} y=x+y$ and $\widehat{x \cdot y}=x \cdot y$. We begin with the latter equality: $\widehat{x \cdot y}=\left(x^{\prime} \widehat{\oplus} y^{\prime}\right)^{\prime}=x \cdot y$. Concerning the former, we have that $x \widehat{+} y=\left(x^{\prime} \widehat{\oplus} y\right)^{\prime} \widehat{\oplus} y=\alpha((\alpha(x \cdot \alpha(y))) \cdot \alpha(y))=x+y$ by Lemma 3-(e).

As a corollary of the representation of basic algebras as Lukasiewicz near semirings, we get the one-to-one correspondence between MV-algebras and the variety of commutative Łukasiewicz near semiring. The following results readily follow from Theorems 4 and 5 and the fact that a basic algebra is an MV-algebra if and only if $\oplus$ is associative.

Corollary 3

Let $\mathbf{M}=\left\langle M, \oplus,^{\prime}, 0\right\rangle$ an MV-algebra. Then the structure $\mathbf{R}(\mathbf{M})=\langle B,+, \cdot, \alpha, 0,1\rangle$, where $x+y, x \cdot y$ and $\alpha(x)$ are defined by $\left(x^{\prime} \oplus y\right)^{\prime} \oplus y,\left(x^{\prime} \oplus y^{\prime}\right)^{\prime}, x^{\prime}$, and $1=0^{\prime}$ respectively, is a Łukasiewicz semiring.

Corollary 4

Let $\mathbf{R}=\langle R,+, \cdot, \alpha, 0,1\rangle$ be a Łukasiewicz semiring and let $x \oplus y=\alpha((\alpha(x)+y) \cdot \alpha(y))$. Then $\mathbf{M}(\mathbf{R})=$ $\langle R, \oplus, \alpha, 0\rangle$ is an MV-algebra.

Corollaries above are tightly related to a very similar result in [2], where it is shown that to every MV-algebra corresponds an MV-semiring: a commutative semiring with involution that satisfies the identity (c) in Lemma 3 (in our terminology) and

$$
x+y=\alpha(\alpha(x) \cdot \alpha(\alpha(x) \cdot y)) .
$$

\section{Congruence properties of Lukasiewicz near semirings}

In this section, we discuss several congruence properties of Łukasiewicz near semirings. Recall that an algebra $\mathbf{A}$ is congruence regular if any congruence $\theta \in \operatorname{Con}(\mathbf{A})$ is determined by any of its cosets; namely if $\theta, \phi \in \operatorname{Con}(\mathbf{A})$ and $a \in A$ then

$$
[a]_{\theta}=[a]_{\phi} \text { implies } \theta=\phi .
$$


A variety $\mathcal{V}$ is congruence regular if every member of $\mathcal{V}$ is congruence regular. A theorem due to Csákány shows (see [6] for details) that a variety $\mathcal{V}$ is congruence regular if and only if there exists a set of ternary terms $t_{i}(x, y, z)$ with $i \geq 1$ such that

$$
t_{i}(x, y, z)=z \text { for any } i \text { if and only if } x=y .
$$

An algebra $\mathbf{A}$ is said to be congruence permutable if for any two congruences $\theta, \phi \in \operatorname{Con}(\mathbf{A})$ it holds that $\theta \circ \phi=\phi \circ \theta$.

An algebra $\mathbf{A}$ is congruence distributive if the complete lattice of its congruences is distributive.

A variety $\mathcal{V}$ is congruence permutable (congruence distributive, resp.) if every member of $\mathcal{V}$ is congruence permutable (congruence distributive, resp.).

Finally, an algebra $\mathbf{A}$ is arithmetical if it is both congruence permutable and congruence distributive. A variety $\mathcal{V}$ is arithmetical if each algebra $\mathbf{A} \in \mathcal{V}$ is arithmetical.

It was proven by Mal'cev [17] that congruence permutability is equivalent to the existence of a certain (uniformly defined) term operation. Precisely, a variety $\mathcal{V}$ is congruence permutable if and only if there exists a ternary term operation $p(x, y, z)$ such that the identities

$$
p(x, x, y)=y \text { and } p(x, y, y)=x
$$

hold in $\mathcal{V}$. The term $p$ is usually referred to as a Mal'cev term for $\mathcal{V}$.

Similarly, congruence distributivity is witnessed by the existence of the so-called Jónsson terms. In particular, a variety $\mathcal{V}$ is congruence distributive if there exists a ternary term operation $M(x, y, z)$, for which the identities

$$
M(x, x, y)=M(x, y, x)=M(y, x, x)=x
$$

hold in $\mathcal{V} . M$ is usually referred to as a majority term for $\mathcal{V}$.

THEOREM 7

The variety of Łukasiewicz near semirings is congruence regular, with witness terms:

$$
\begin{aligned}
& t_{1}(x, y, z)=((x \cdot \alpha(y))+(y \cdot \alpha(x))+z ; \\
& t_{2}(x, y, z)=\alpha((x \cdot \alpha(y))+(y \cdot \alpha(x))) \cdot z .
\end{aligned}
$$

Proof. All we need to check is that $t_{1}(x, y, z)=t_{2}(x, y, z)=z$ if and only if $x=y$. Suppose that $x=y$; then $t_{1}(x, x, z)=\left((x \cdot \alpha(x))+(x \cdot \alpha(x))+z=(0+0)+z=0+z=z\right.$. On the other hand $t_{2}(x, x, z)=\alpha((x$. $\alpha(x))+(x \cdot \alpha(x))) \cdot z=\alpha(0+0) \cdot z=1 \cdot z=z$. For the converse, suppose $t_{1}(x, y, z)=t_{2}(x, y, z)=z$, which, setting $a=(x \cdot \alpha(y))+(y \cdot \alpha(x))$, reads

$$
\begin{aligned}
& a+z=z \\
& \alpha(a) \cdot z=z
\end{aligned}
$$

Equation (4.1) above implies that $a \leq z$, hence $\alpha(z) \leq \alpha(a)$. We now claim that $a=0$. Indeed

$$
\begin{aligned}
& a=\alpha(\alpha(a)) \\
& =\alpha(\alpha(a)+\alpha(z)) \\
& =\alpha(\alpha(a) \cdot z) \cdot z \\
& =\alpha(z) \cdot z \\
& =0
\end{aligned}
$$




\section{Near semirings}

Therefore $a=(x \cdot \alpha(y))+(y \cdot \alpha(x))=0$. Since $\langle R,+\rangle$ is a join-semilattice with 0 as least element, $(x \cdot \alpha(y))+(y \cdot \alpha(x))=0$ implies that $x \cdot \alpha(y)=0$ and $y \cdot \alpha(x)=0$. Using Lemma 4, we get $x \leq y$ and $y \leq x$, proving that $x=y$ as desired.

THEOREM 8

The variety of Łukasiewicz near semirings is arithmetical, with witness Mal'cev term

$$
p(x, y, z)=\alpha((\alpha(x \cdot \alpha(y)) \cdot \alpha(z))+(\alpha(z \cdot \alpha(y)) \cdot \alpha(x))) .
$$

Proof. We first show that the term $p(x, y, z)$ is a Mal'cev term for the variety of Łukasiewicz near semiring: $p(x, y, y)=x$ and $p(x, x, y)=y$.

$$
\begin{aligned}
& p(x, y, y)=\alpha((\alpha(x \cdot \alpha(y)) \cdot \alpha(y))+(\alpha(y \cdot \alpha(y)) \cdot \alpha(x))) \\
& =\alpha((\alpha(x+y)+\alpha(x)) \\
& =\alpha(\alpha(x))=x
\end{aligned}
$$

Similarly,

$$
\begin{aligned}
& p(x, x, y)=\alpha((\alpha(x \cdot \alpha(x)) \cdot \alpha(y))+(\alpha(y \cdot \alpha(x)) \cdot \alpha(x))) \\
& =\alpha((\alpha(y)+\alpha(x+y)) \\
& =\alpha(\alpha(y))=y
\end{aligned}
$$

Therefore, the variety of Łukasiewicz near semirings is congruence permutable. Moreover, the following ternary term

$$
M(x, y, z)=\alpha(\alpha(x)+\alpha(y))+\alpha(\alpha(y)+\alpha(z))+\alpha(\alpha(z)+\alpha(x))
$$

is a majority term for the variety of Łukasiewicz near semiring. A simple calculation shows that $M(x, x, y)=M(x, y, x)=M(y, x, x)=x$. This proves that the variety considered is also congruence distributive, hence by definition it is arithmetical as claimed.

\section{Orthomodular lattices as near semirings}

Orthomodular lattices were introduced in 1936 by Birkhoff and von Neumann as an algebraic account of the logic of quantum mechanics. A detailed discussion can be found in [3, 15]. The aim of this section is to provide a representation of orthomodular lattices as involutive near semirings.

Let us briefly recall that an orthomodular lattice (OML, for short) is an algebra $\mathbf{L}=\left\langle L, \vee, \wedge,,^{\prime}, 0,1\right\rangle$ of type $\langle 2,2,1,0,0\rangle$ such that $\langle L, \vee, \wedge, 0,1\rangle$ is a bounded lattice, ' is an orthocomplementation, i.e. $x \wedge x^{\prime}=0, x \vee x^{\prime}=1$. Furthermore ' is an involutive, antitone map ( $x \leq y$ implies $\left.y^{\prime} \leq x^{\prime}\right)$ that satisfies the so called orthomodular law:

$$
x \leq y \Rightarrow y=x \vee\left(y \wedge x^{\prime}\right)
$$

The orthomodular law can be equivalently expressed by the identity

$$
(x \vee y) \wedge\left(x \vee(x \vee y)^{\prime}\right)=x,
$$


which, in turn, is equivalent to the dual form:

$$
(x \wedge y) \vee\left(y \wedge(x \wedge y)^{\prime}\right)=y .
$$

In the next lemma, we recap some basic facts relative to OMLs which will be useful in what follows. Let $a, b$ two elements of an OML $\mathbf{L}$, we say that $a$ and $b$ commute (in symbols $a C b$ ) iff $a=(a \wedge b) \vee\left(a \wedge b^{\prime}\right)$. For the proof of Lemma 5, see [3] or [15].

LEMMA 5

Let $\mathbf{L}$ an orthomodular lattice and $a, b, c \in L$. Then

(i) If $a C b$ then $b C a$.

(ii) If $a \leq b$ then $a C b$.

(iii) If $a C b$ then $a C b^{\prime}$.

(iv) If two elements among $a, b, c$ commutes with the third, then $(a \vee b) \wedge c=(a \wedge c) \vee(a \wedge c)$ and $(a \wedge b) \vee c=(a \vee c) \wedge(b \vee c)$.

In the previous section, we introduced Łukasiewicz near semirings to represent basic algebras. Here, to provide a similar representation of OMLs, we will consider orthomodular near semirings.

DeFINITION 4

An orthomodular near semiring $\mathbf{R}$ is a Łukasiewicz near semiring that fulfills the following identity:

$$
x=x \cdot(x+y) .
$$

The next lemma shows some basic properties of orthomodular near semirings.

LEMMA 6

Let $\mathbf{R}$ be an orthomodular near semiring. Then:

(a) $x \cdot x=x$;

(b) $x=x \cdot \alpha((\alpha(y \cdot \alpha(x)) \cdot \alpha(x))$;

(c) $x+\alpha(x)=1$;

(d) If $x \leq y$ then $x \cdot y=y$.

Proof. (a) Straightforward, by setting $y=0$ (or also $x=y$ ) in Equation (5.4).

(b) follows directly using Equation (5.4) and Lemma 3-(e).

(c) By Lemma 3-(e), we have $x+\alpha(x)=\alpha(\alpha(x \cdot x) \cdot x)=\alpha(\alpha(x) \cdot x)=1$, where we have used (a).

(d) Let $a \leq b$, then $a+b=b$. Therefore $a=a \cdot(a+b)=a \cdot b$.

We first show that an orthomodular near semiring can always be obtained out of an OML.

\section{THEOREM 9}

Let $\mathbf{L}=\left\langle L, \vee, \wedge,{ }^{\prime}, 0,1\right\rangle$ an orthomodular lattice and define multiplication via the so-called Sasaki projection: $x \cdot y:=\left(x \vee y^{\prime}\right) \wedge y$. Then $\mathbf{R}(\mathbf{L})=\left\langle L,+, \cdot{ }^{\prime}, 0,1\right\rangle$ is an orthomodular near semiring, where $x+y=x \vee y$.

Proof. It is evident that $\langle L, \vee, 0\rangle$ is a commutative, idempotent monoid. Furthermore, $x \cdot 1=\left(x \vee 1^{\prime}\right) \wedge$ $1=(x \vee 0) \wedge 1=x$, and $1 \cdot x=\left(1 \vee x^{\prime}\right) \wedge x=1 \wedge x=x$. Therefore $\langle R, \cdot, 1\rangle$ is a groupoid with 1 as neutral element. To prove right distributivity we make use of Lemma 5. Upon observing that $z^{\prime} \leq x \vee z^{\prime}$, 


\section{Near semirings}

$z^{\prime} \leq y \vee z^{\prime}$, we have that $z^{\prime}$ commutes (in the sense of Lemma 5) with both $x \vee z^{\prime}$ and $y \vee y^{\prime}$, therefore $z$ does. For this reason we get:

$$
\begin{aligned}
& (x \vee y) \cdot z=\left((x \vee y) \vee z^{\prime}\right) \wedge z \quad \text { (Definition) } \\
& =\left(\left(x \vee z^{\prime}\right) \vee\left(y \vee z^{\prime}\right)\right) \wedge z \quad \text { (Lattice prop.) } \\
& =\left(\left(x \vee z^{\prime}\right) \wedge z\right) \vee\left(\left(y \vee z^{\prime}\right) \wedge z\right) \quad \text { (Lemma 5-(iv)) } \\
& =(x \cdot z) \vee(y \cdot z) \text {. }
\end{aligned}
$$

It is not difficult to check that 0 annihilates multiplication. Indeed, $x \cdot 0=\left(x \vee 0^{\prime}\right) \wedge 0=0$ and $0 \cdot x=$ $\left(0 \vee x^{\prime}\right) \wedge x=x^{\prime} \wedge x=0$. We now show that $\mathbf{R}(\mathbf{L})$ is Łukasiewicz near semiring (see Definition 3 ). First let us observe that:

$$
\begin{array}{ll}
\left(x \cdot y^{\prime}\right)^{\prime} \cdot y^{\prime}=\left(\left((x \vee y) \wedge y^{\prime}\right)^{\prime} \vee y\right) \wedge y^{\prime} & \text { (Definition) } \\
=\left(\left((x \vee y)^{\prime} \vee y\right) \vee y\right) \wedge y^{\prime} & \text { (De Morgan) } \\
=\left((x \vee y)^{\prime} \vee y\right) \wedge y^{\prime} & \text { (Ass., Idem.) }
\end{array}
$$

Reasoning similarly one gets $\left(y \cdot x^{\prime}\right)^{\prime} \cdot x^{\prime}=\left((x \vee y)^{\prime} \vee x\right) \wedge x^{\prime}$.

Simply observing that $x \leq x \vee y$ and applying the orthomodular law, we have $x \vee y=x \vee((x \vee y) \wedge$ $\left.x^{\prime}\right)$. Therefore,

$$
\begin{aligned}
& (x \vee y)^{\prime}=\left(x \vee\left((x \vee y) \wedge x^{\prime}\right)\right)^{\prime} \\
& =x^{\prime} \wedge\left((x \vee y) \wedge x^{\prime}\right)^{\prime} \quad \text { (De Morgan) } \\
& =\left((x \vee y)^{\prime} \vee x\right) \wedge x^{\prime} \quad \text { (Comm.) } \\
& =\left(y \cdot x^{\prime}\right)^{\prime} \cdot x^{\prime}
\end{aligned}
$$$$
=x^{\prime} \wedge\left((x \vee y)^{\prime} \vee x\right) \quad \text { (De Morgan) }
$$

Analogously, using the fact that $y \leq x \vee y$ one gets, by the orthomodular law, that $(x \vee y)^{\prime}=((x \vee$ $\left.y)^{\prime} \vee y\right) \wedge y^{\prime}=\left(x \cdot y^{\prime}\right)^{\prime} \cdot y^{\prime}$. Therefore $\left(x \cdot y^{\prime}\right)^{\prime} \cdot y^{\prime}=\left(y \cdot x^{\prime}\right)^{\prime} \cdot x^{\prime}$ as claimed. We finally check that also Equation (5.4) holds. This is a simple consequence of the orthomodular law: $x \cdot(x+y)=\left(x \vee(x \vee y)^{\prime}\right) \wedge$ $(x \vee y)=x$ by Equation (5.2). Therefore, $\mathbf{R}(\mathbf{L})=\left\langle L, \vee, \cdot,{ }^{\prime}, 0,1\right\rangle$ is an orthomodular near semiring. ${ }^{4}$

We can also prove the converse, stating a correspondence between orthomodular lattices and orthomodular near semirings.

THEOREM 10

Let $\mathbf{R}$ be an orthomodular near semiring. Setting $x \vee y=x+y, x^{\prime}=\alpha(x)$, and then defining $x \wedge y=$ $\left(x^{\prime} \vee y^{\prime}\right)^{\prime}$, then $\mathbf{L}(\mathbf{R})=\left\langle R, \vee, \wedge,{ }^{\prime}, 0,1\right\rangle$ is an orthomodular lattice.

PRoof. Since $\mathbf{R}$ is integral we know that $\langle R,+\rangle$ is a join-semilattice with 1 as top element, and consequently $\langle R, \vee\rangle$ is. On the other hand, since $\alpha$ is an antitone involution then $\langle R, \wedge\rangle$ is a meetsemilattice with 0 as least element. As meet and join are defined dually, $\langle R, \vee, \wedge, 0,1\rangle$ is a bounded lattice. Furthermore, $x \vee x^{\prime}=1$ is guaranteed by Lemma 6 and thus it follows that $x \wedge x^{\prime}=0$.

\footnotetext{
${ }^{4}$ Notice that $\langle L, \vee, \cdot\rangle$, in general, is not a lattice.
} 
We are left with the task of showing that the orthomodular law holds too. So, suppose $a \leq b$, then

$$
\begin{aligned}
& a \vee\left(b \wedge a^{\prime}\right)=a+\alpha(\alpha(b)+a) \\
& =a+\alpha(a+\alpha(b)) \quad \text { (Comm.) } \\
& =a+(\alpha(a \cdot b) \cdot b) \quad \text { (Lemma 3) } \\
& =(a \cdot b)+(\alpha(a) \cdot b) \quad \text { (Lemma 6-(d)) } \\
& =(a+\alpha(a)) \cdot b \quad \text { (Distr.) } \\
& =1 \cdot b=b \text {. }
\end{aligned}
$$

This allows to conclude that $\mathbf{L}(\mathbf{R})$ is an orthomodular lattice.

The theorems above have shown how to get an orthomodular lattice out of an orthomodular semirings and viceversa. In other words, there are maps $f, g$, from the variety of orthomodular lattices to the variety of orthomodular semirings and from the variety of orthomodular near semirings to the variety of orthomodular lattices, respectively, assigning to any OML an orthomodular semiring, and vice versa. We now show that:

\section{THEOREM 11}

The maps $f$ and $g$ are mutually inverse: $\mathbf{L}=\mathbf{L}(\mathbf{R}(\mathbf{L}))$ and $\mathbf{R}=\mathbf{R}(\mathbf{L}(\mathbf{R}))$.

Proof. Let $\mathbf{L}\left\langle L, \vee, \wedge,{ }^{\prime}, 0,1\right\rangle$ be an orthomodular lattice. It follows from Theorem 9 that $\mathbf{R}(\mathbf{L})$ is an orthomodular near semiring, and from Theorem 10 that the structure $\mathbf{L}(\mathbf{R}(\mathbf{L}))=\left\langle L, \bar{v}, \bar{\wedge},{ }^{\prime}, 0,1\right\rangle$ is an orthomodular lattice. It is straightforward to check that the involutions on $\mathbf{L}(\mathbf{R}(\mathbf{L}))$ and $\mathbf{L}$ coincide, as well as $x \bar{\vee} y=x \vee y$. Therefore we also have that $x \bar{\wedge} y=\left(x^{\prime} \bar{\vee} y^{\prime}\right)^{\prime}=\left(x^{\prime} \vee y^{\prime}\right)^{\prime}=x \wedge y$. So $\mathbf{L}=\mathbf{L}(\mathbf{R}(\mathbf{L}))$. On the other hand, by Theorems 9 and 10 we obtain that the structure $\mathbf{R}(\mathbf{L}(\mathbf{R}))=\langle R, \widehat{+}, \widehat{\cdot}, \widehat{\alpha}, 0,1\rangle$ is an orthomodular near semiring. Again it is straightforward to check that $\widehat{\alpha}(x)=\alpha(x)$ and $x \widehat{+} y=x+y$. It is less evident that $\widehat{x \cdot y}=x \cdot y$. Indeed:

$$
\begin{aligned}
& \widehat{x \cdot y}=\left(x \vee y^{\prime}\right) \wedge y \\
& =\left(\left(x \vee y^{\prime}\right)^{\prime} \vee y^{\prime}\right)^{\prime} \\
& =\alpha(\alpha(x+\alpha(y))+\alpha(y)),
\end{aligned}
$$

where $\vee, \wedge$ and ' are join, meet and complementation, respectively, of the orthomodular lattice $\mathbf{L}(\mathbf{R})$. We are finally left with showing that $\alpha(\alpha(x+\alpha(y))+\alpha(y))=x \cdot y$.

Our first move is to prove that $\alpha(\alpha(x+\alpha(y))+\alpha(y))=y \cdot(x+\alpha(y))$.

$$
\begin{aligned}
& \alpha(\alpha(x+\alpha(y))+\alpha(y))=\alpha(\alpha(y)+\alpha(x+\alpha(y))) \\
& =\alpha(\alpha(y) \cdot(x+\alpha(y))) \cdot(x+\alpha(y)) \\
& =\alpha(\alpha(y)) \cdot(x+\alpha(y)) \\
& =y \cdot(x+\alpha(y)) .
\end{aligned}
$$

Moreover,

$$
\begin{array}{ll}
\alpha((x \cdot y) \cdot y) \cdot y=\alpha((x \cdot y)+\alpha(y)) & (\text { Lemma 3) } \\
=\alpha(\alpha(y)+(x \cdot y)) & (\text { Comm. }) \\
=\alpha(\alpha(y) \cdot \alpha(x \cdot y)) \cdot \alpha(x \cdot y) & (\text { Lemma 3) }
\end{array}
$$


16 Near semirings

$$
\begin{array}{ll}
=\alpha(\alpha(y)) \cdot \alpha(x \cdot y) & \text { (by Lemma } 6, \text { since } \alpha(y) \leq \alpha(x \cdot y)) \\
=y \cdot \alpha(x \cdot y) . &
\end{array}
$$

Using the derivation above, which we will refer to as $(\star)$, we finally prove our claim:

$$
\begin{aligned}
& y \cdot(x+\alpha(y))=y \cdot \alpha(\alpha(x \cdot y) \cdot y) \quad \text { (Lemma 3) } \\
& =\alpha((\alpha(x \cdot y) \cdot y) \cdot y) \cdot y \\
& =\alpha(\alpha(x \cdot y) \cdot y) \cdot y \quad \text { (by Lemma 6, since } \alpha(x \cdot y) \cdot y \leq y) \\
& =(x+\alpha(y)) \cdot y \quad \text { (Lemma 3) } \\
& =(x \cdot y)+(\alpha(y) \cdot y) \quad \text { (Right Distr.) } \\
& =x \cdot y \text {. (Lemma 3) }
\end{aligned}
$$

\section{Central elements and decomposition}

The aim of this section is to give a characterization of the central elements and consequently some decomposition theorems for the variety of integral involutive near semirings. Such results apply to both the variety of Łukasiewicz near semirings and the orthomodular near semirings as the they are both integral. The section relies on the ideas developed in [18] and [16] on the general theory of Church algebras. ${ }^{5}$

The notion of Church algebra is based on the simple observation that many well-known algebras, including Heyting algebras, rings with unit and combinatory algebras, possess a ternary term operation $q$, satisfying the equations: $q(1, x, y)=x$ and $q(0, x, y)=y$. The term operation $q$ simulates the behaviour of the if-then-else connective and, surprisingly enough, this yields rather strong algebraic properties.

An algebra $\mathbf{A}$ of type $v$ is a Church algebra if there are term definable elements $0^{\mathbf{A}}, 1^{\mathbf{A}} \in A$ and a ternary term operation $q^{\mathbf{A}}$ s.t., for all $a, b \in A, q^{\mathbf{A}}\left(1^{\mathbf{A}}, a, b\right)=a$ and $q^{\mathbf{A}}\left(0^{\mathbf{A}}, a, b\right)=b$. A variety $\mathcal{V}$ of type $v$ is a Church variety if every member of $\mathcal{V}$ is a Church algebra with respect to the same term $q(x, y, z)$ and the same constants 0,1 .

Taking up a suggestion from Diego Vaggione [20], we say that an element $e$ of a Church algebra $\mathbf{A}$ is central if the pair $(\theta(e, 0), \theta(e, 1))$ is a pair of factor congruences on $\mathbf{A}$. A central element $e$ is non-trivial when $e \notin\{0,1\}$. We denote the set of central elements of $\mathbf{A}$ (the centre) by $\operatorname{Ce}(A)$.

Setting

$$
x \wedge y=q(x, y, 0), x \vee y=q(x, 1, y) x^{*}=q(x, 0,1)
$$

we can report the following general result on Church algebras:

THEOREM 12 ([18])

Let $\mathbf{A}$ be a Church algebra. Then

$$
\mathrm{Ce}(\mathbf{A})=\left\langle\mathrm{Ce}(A), \wedge, \vee,^{*}, 0,1\right\rangle
$$

is a Boolean algebra which is isomorphic to the Boolean algebra of factor congruences of $\mathbf{A}$.

\footnotetext{
${ }^{5}$ Diverse applications of this theory can be found in [4, 14].
} 
If $\mathbf{A}$ is a Church algebra of type $v$ and $e \in A$ is a central element, then we define $\mathbf{A}_{e}=\left(A_{e}, g_{e}\right)_{g \in v}$ to be the $v$-algebra defined as follows:

$$
A_{e}=\{e \wedge b: b \in A\} ; \quad g_{e}(e \wedge \bar{b})=e \wedge g(e \wedge \bar{b}),
$$

where $\bar{b}$ denotes the a n-tuple $b_{1}, \ldots, b_{n}$ and $e \wedge \bar{b}$ is an abbreviation for $e \wedge b_{1}, \ldots, e \wedge b_{n}$.

By [16, Theorem 4], we have that:

\section{THEOREM 13}

Let $\mathbf{A}$ be a Church algebra of type $v$ and $e$ be a central element. Then we have:

(1) For every $n$-ary $g \in v$ and every sequence of elements $\bar{b} \in A^{n}, e \wedge g(\bar{b})=e \wedge g(e \wedge \bar{b})$, so that the function $h: A \rightarrow A_{e}$, defined by $h(b)=e \wedge b$, is a homomorphism from $\mathbf{A}$ onto $\mathbf{A}_{e}$.

(2) $\mathbf{A}_{e}$ is isomorphic to $\mathbf{A} / \theta(e, 1)$. It follows that $\mathbf{A}=\mathbf{A}_{e} \times \mathbf{A}_{e^{\prime}}$ for every central element $e$, as in the Boolean case.

Proposition 1

The class of intergral involutive near semirings is a Church variety, as witnessed by the term:

$$
q(x, y, z)=(x \cdot y)+(\alpha(x) \cdot z) .
$$

Proof. Suppose $\mathbf{R}$ is an integral involutive near semiring and $a, b \in R$. Then $q(1, a, b)=(1 \cdot a)+$ $(\alpha(1) \cdot b)=a+(0 \cdot b)=a+0=a$. and $q(0, a, b)=(0 \cdot a)+(\alpha(0) \cdot b)=0+(1 \cdot b)=0+b=b$.

Since both the varieties of Łukasiewicz and orthomodular near semirings are subvarieties of integral involutive near semiring, it follows that both of them are Church varieties. In this section, we apply the theory of Church algebras to the more general class of integral involutive near semirings. According with the results in [18, Proposition 3.6], in a Church variety central elements are amenable to a very general description.

Proposition 2

If $\mathbf{A}$ is a Church algebra of type $v$ and $e \in A$, the following conditions are equivalent:

(1) e is central;

(2) for all $a, b, \vec{a}, \vec{b} \in A$ :

a) $q(e, a, a)=a$,

b) $q(e, q(e, a, b), c)=q(e, a, c)=q(e, a, q(e, b, c))$,

c) $q(e, f(\vec{a}), f(\vec{b}))=f\left(q\left(e, a_{1}, b_{1}\right), \ldots, q\left(e, a_{n}, b_{n}\right)\right)$, for every $f \in v$,

d) $q(e, 1,0)=e$.

In case $\mathbf{A}$ is an integral involutive near semiring, condition (a) reduces to

$$
(e \cdot a)+(\alpha(e) \cdot a)=a .
$$

Conditions (b) read

$$
\begin{aligned}
& (e \cdot((e \cdot a)+(\alpha(e) \cdot b)))+(\alpha(e) \cdot c))=(e \cdot a)+(\alpha(e) \cdot c), \\
& (e \cdot a)+(\alpha(e) \cdot c)=(e \cdot a)+(\alpha(e) \cdot((e \cdot b)+(\alpha(e) \cdot c))) .
\end{aligned}
$$

Condition (c), whenever $f$ is the constant 0 , expresses a property that holds for every element: $(e \cdot 0)+(\alpha(e) \cdot 0)=0$. On the other hand, if $f$ coincides with the nullary operation 1 , we obtain (for a 


\section{Near semirings}

central element $e$ )

$$
q(e, 1,1)=(e \cdot 1)+(\alpha(e) \cdot 1)=e+\alpha(e)=1 .
$$

If $f$ coincides with the involution, (c) reads

$$
(e \cdot \alpha(a))+(\alpha(e) \cdot \alpha(b))=\alpha((e \cdot a)+(\alpha(e) \cdot b)) .
$$

Whenever $f$ is + , we obtain:

$$
(e \cdot(a+c))+(\alpha(e) \cdot(b+d))=((e \cdot a)+(\alpha(e) \cdot b))+((e \cdot c)+(\alpha(e) \cdot d))
$$

this, by the associativity of the sum, is equal to

$$
((e \cdot a)+(e \cdot c))+((\alpha(e) \cdot b)+(\alpha(e) \cdot d))
$$

which is a sort of distributivity restricted to central elements. Whenever $f$ is the multiplication, this condition simplifies to

$$
(e \cdot(a \cdot c))+(\alpha(e) \cdot(b \cdot d))=((e \cdot a)+(\alpha(e) \cdot b)) \cdot((e \cdot c)+(\alpha(e) \cdot d)) .
$$

Condition (d) expresses a general property that holds true for every element: $(e \cdot 1)+(\alpha(e) \cdot 0)=$ $e+0=e$. We have just seen in Proposition 2 that, in Church algebras, central elements can be described by means of identities. This, in fact, will be very useful in proving the results in this section. However, we aim to show that the axiomatization of central elements can be streamlined to a minimal set (see Appendix 5) of two identities only. The next lemma introduces some results which are very useful to prove the minimality of such an axiomatization.

LEMMA 7

Let $\mathbf{R}$ be an integral involutive near semiring, and $e \in R$ an element that satisfies the following identities:

(1) $(e \cdot \alpha(x))+(\alpha(e) \cdot \alpha(y))=\alpha((e \cdot x)+(\alpha(e) \cdot y))$;

(2) $(e \cdot(x \cdot z))+(\alpha(e) \cdot(y \cdot u))=((e \cdot x)+(\alpha(e) \cdot y)) \cdot((e \cdot z)+(\alpha(e) \cdot u))$.

Then $e$ satisfies the following:

(i) $(e \cdot x)+\alpha(e)=x+\alpha(e)$;

(ii) $e \cdot(e \cdot x)=e \cdot x=(e \cdot x) \cdot e$;

(iii) $e \cdot \alpha(e)=0$;

(iv) $e \cdot x=x \cdot e$;

(v) $e \cdot(x+y)=(e \cdot x)+(e \cdot y)$;

(vi) if $x \leq y$ then $e \cdot x \leq e \cdot y$;

(vii) $e \cdot(\alpha(e) \cdot x)=0$.

Proof. (i) Since $e \leq 1$, then $e \cdot x \leq 1 \cdot x=x$. Therefore $(e \cdot x)+\alpha(e) \leq x+\alpha(e)$. For the converse, first notice that, as $e \cdot \alpha(x) \leq \alpha(x)$, then $x \leq \alpha(e \cdot \alpha(x))=(e \cdot x)+\alpha(e)$, where the last equality is obtained by setting $y=1$ in identity (1) (and the fact that $\alpha$ is an involution).

(ii) The first equality readily follows from (2) upon setting $y=u=0$ and $x=1$, while the second by setting $y=u=0$ and $z=1$. 
(iii) can be derived by setting $x=u=1$ and $y=z=0$ in identity (2).

(iv)

$$
\begin{aligned}
& e \cdot x=(e \cdot x) \cdot e \\
& =((e \cdot x) \cdot e)+(\alpha(e) \cdot e) \\
& =((e \cdot x)+\alpha(e)) \cdot e \\
& =(x+\alpha(e)) \cdot e \\
& =(x \cdot e)+(\alpha(e) \cdot e) \\
& =(x \cdot e)+0 \\
& =x \cdot e .
\end{aligned}
$$

(v)

$$
\begin{aligned}
& e \cdot(x+y)=(x+y) \cdot e \\
& =(x \cdot e)+(y \cdot e) \\
& =(e \cdot x)+(e \cdot y)
\end{aligned}
$$

(vi) Let $x \leq y$, i.e. $x+y=y$. Then $e \cdot y=e \cdot(x+y)=(e \cdot x)+(e \cdot y)$, i.e. $e \cdot x \leq e \cdot y$.

(vii) In case $y=u=0$, in condition (3), we obtain: $e \cdot(x \cdot z)=(e \cdot x) \cdot(x \cdot y)$. If, moreover, $x=\alpha(e)$, we obtain that $e \cdot(\alpha(e) \cdot z)=(e \cdot \alpha(e)) \cdot(e \cdot z)=0$, by (iii).

We now put Lemma 7 to good use and prove that, in an involutive near semiring, central elements are neatly characterized by two simple equations.

THEOREM 14

Let $\mathbf{R}$ be an involutive near semiring. Then an element $e \in R$ is central if and only if it satisfies the following equations for any $x, y, z, u \in R$ :

(1) $(e \cdot \alpha(x))+(\alpha(e) \cdot \alpha(y))=\alpha((e \cdot x)+(\alpha(e) \cdot y))$;

(2) $(e \cdot(x \cdot z))+(\alpha(e) \cdot(y \cdot u))=((e \cdot x)+(\alpha(e) \cdot y)) \cdot((e \cdot z)+(\alpha(e) \cdot u))$.

Proof. $(\Rightarrow)$ If $e$ is a central element then (1), (2) hold by Proposition 2.

$(\Leftarrow)$ Using again Proposition 2, and identities (1) and (2), we have to derive Equations (6.2), (6.3), (6.4), (6.5) and (6.7). We start by deriving (6.5): upon setting $x=y=0$, identity (1) reads: $e+\alpha(e)=\alpha(0)=1$. Using (6.5), we obtain (6.2) as follows

$$
\begin{aligned}
& (e \cdot x)+(\alpha(e) \cdot x)=(e+\alpha(e)) \cdot x \\
& =1 \cdot x \\
& =x .
\end{aligned}
$$

Equation (6.7) immediately follows from the associativity of the sum and the fact that $e \cdot(x+y)=$ $(e \cdot x)+(e \cdot y)$ from Lemma 7. In order to prove (6.3) and (6.4) we use some auxiliary facts stated in Lemma 7.

$$
\begin{aligned}
& (e \cdot((e \cdot a)+(\alpha(e) \cdot b)))+(\alpha(e) \cdot c)= \\
& =(e \cdot(e \cdot a))+(e \cdot(\alpha(e) \cdot b))+(\alpha(e) \cdot c) \quad(\text { Lemma 7.(v)) }
\end{aligned}
$$




$$
\begin{aligned}
& =(e \cdot a)+(e \cdot(\alpha(e) \cdot b))+(\alpha(e) \cdot c) \\
& =(e \cdot a)+0+(\alpha(e) \cdot c) \\
& =(e \cdot a)+(\alpha(e) \cdot c) .
\end{aligned}
$$

With a slight modification of the reasoning above one can derive condition (6.4).

The next proposition yields a more informative version of the general result stated in Theorem 12 .

Proposition 3

Let $\mathbf{R}$ be an integral involutive near semiring and $\operatorname{Ce}(R)$ the set of central elements of $\mathbf{R}$. Then $\mathrm{Ce}(\mathbf{R})=\langle\mathrm{Ce}(R),+, \cdot, \alpha, 0,1\rangle$ is a Boolean algebra.

Proof. By Theorem 12, $\mathrm{Ce}(\mathbf{R})=\left\langle\mathrm{Ce}(R), \wedge, \vee,{ }^{*}, 0,1\right\rangle$ is a Boolean algebra, where $\wedge, \vee,{ }^{*}$ are defined as follows

$$
x \wedge y=q(x, y, 0), x \vee y=q(x, 1, y) x^{*}=q(x, 0,1)
$$

Using this result, we just check that, for central elements, $\wedge, \vee,{ }^{*}$ coincide with $\cdot,+, \alpha$, respectively. We can easily obtain that $x \wedge y=q(x, y, 0)=(x \cdot y)+(\alpha(x) \cdot 0)=x \cdot y$, and $x^{*}=q(x, 0,1)=(x \cdot 0)+(\alpha(x)$. $1)=\alpha(x)$.

It only remains to show that $x+y=\alpha(\alpha(x) \cdot \alpha(y))$. Notice first that, by Equation (6.6), with $a=0$, $b=y^{\prime}$ and $e=x$ (this is legitimated by the fact that we are only concerned with central elements), we have

$$
x+(\alpha(x) \cdot y)=\alpha(\alpha(x) \cdot \alpha(y))
$$

Since, for central elements, multiplication coincides with the Boolean meet, we have that $\alpha(x)$. $\alpha(y) \leq \alpha(x)$ and $\alpha(x) \cdot \alpha(y) \leq \alpha(y)$. As $\alpha$ is antitone, $x \leq \alpha(\alpha(x) \cdot \alpha(y))$ and $y \leq \alpha(\alpha(x) \cdot \alpha(y))$, which implies that $x+y \leq \alpha(\alpha(x) \cdot \alpha(y))+\alpha(\alpha(x) \cdot \alpha(y))=\alpha(\alpha(x) \cdot \alpha(y))$. For the converse, $\alpha(x) \cdot y \leq y$, so $x+(\alpha(x) \cdot y) \leq x+y$, i.e. $\alpha(\alpha(x) \cdot \alpha(y)) \leq x+y$, by $(\dagger)$. This proves that $x+y=x \vee y$.

From the previous proposition we have that if $\mathbf{R}$ is an integral involutive near semiring and $e$ is a central element, then $\alpha(e)$ is also central. Our next step will be proving a decomposition theorem for involutive intergral near semiring. Let $e$ be a central element of an integral involutive near semiring $\mathbf{R}$, and set

$$
[0, e]=\{x: x \leq e\}
$$

A complementation can be naturally defined on $[0, e]$ by setting $x^{e}=e \cdot \alpha(x)$. Then, upon considering the algebra $[\mathbf{0}, \mathbf{e}]=\left\langle[0, e],+, \cdot,{ }^{e}, 0, e\right\rangle$, we can prove the following:

THEOREM 15

Let $\mathbf{R}$ an integral involutive near semiring and $e$ a central element of $\mathbf{R}$. Then $\mathbf{R} \cong[\mathbf{0}, \mathbf{e}] \times\left[\mathbf{0}, \mathbf{e}^{\prime}\right]$.

Proof. As $\mathbf{R}$ is a Church algebra, it satisfies Theorem 13, hence all we have to prove reduce to the following:

(1) $R_{e}=[0, e]$

(2) for $x, y \leq e, x+y=e \wedge(x+y), x \cdot y=e \wedge(x \cdot y)$ and $x^{e}=e \wedge \alpha(x)$. 
(1) Suppose $x \in R_{e}$, i.e. $x=e \wedge b$ for some $b \in R$. By definition of $\wedge, e \wedge b=q(e, b, 0)=(e \cdot b)+(\alpha(e)$. $0)=e \cdot b$. Now, as $b \leq 1$, by Lemma 7 we have that $e \cdot b \leq e \cdot 1=e$, i.e. $x \in[0, e]$, proving $R_{e} \subseteq[0, e]$. For the converse, suppose $x \in[0, e]$, i.e. $x \leq e$. We want to find an element $b \in R$ such that $x=e \wedge b$. First notice that, under the assumption that $e$ is central, it follows by Theorem 14 and Lemma 7 that $\alpha(e) \cdot x=0$, which we use to prove that

$$
\begin{aligned}
& 0=\alpha(e) \cdot e \\
& =\alpha(e) \cdot(e+x) \\
& =(\alpha(e) \cdot e)+(\alpha(e) \cdot x) \\
& =0+\alpha(e) \cdot x \\
& =\alpha(e) \cdot x .
\end{aligned}
$$

We use the fact above to show that $e \cdot x=x$. Since, by equation (6.5), $1=e+\alpha(e)$, we have that $x=$ $(e+\alpha(e)) \cdot x=(e \cdot x)+(\alpha(e) \cdot x)=(e \cdot x)+0=e \cdot x$. Remembering that $e \wedge b=q(e, b, 0)=(e \cdot b)+(\alpha(e)$. $0)=e \cdot b$ and setting $b=x+\alpha(e)$ we get

$$
\begin{aligned}
& e \wedge b=e \cdot b \\
& =e \cdot(x+\alpha(e)) \\
& =(e \cdot x)+(e \cdot \alpha(e)) \\
& =(e \cdot x)+0 \\
& =e \cdot x=x .
\end{aligned}
$$

Therefore, $x$ can be expressed as the meet of $e$ with an element of $R$, showing that $[0, e] \subseteq R_{e}$.

(2) In this part of the proof we make use of the following facts

$$
x \wedge y=q(x, y, 0)=x \cdot y \text { and if } x \leq e, \text { then } e \cdot x=x .
$$

Let $x, y \leq e$. Then $e \wedge(x+y)=e \cdot(x+y)=x+y$. Similarly, $e \wedge(x \cdot y)=e \cdot(x \cdot y)=x \cdot y$. Finally $x^{e}=e \wedge \alpha(e)=e \cdot \alpha(e)$

Taking advantage from the fact that, in a Church algebra, central elements are equationally characterizable (Proposition 2 and Theorem 14), we can prove the following:

Proposition 4

Let $\mathbf{R}$ be a involutive integral near semiring, $e \in \mathrm{Ce}(\mathbf{R})$ and $c \in R_{e}$. Then

$$
c \in \mathrm{Ce}(R) \Leftrightarrow c \in \operatorname{Ce}\left(R_{e}\right)
$$

Proof. $(\Rightarrow)$ By Theorem 14, central elements are described by equations. Furthermore, by Theorem $13, h: \mathbf{R} \rightarrow \mathbf{R}_{e}$ is an onto homomorphism such that for every $x \in R_{e}, h(x)=x$. The fact that equations are preserved by homomorphisms yields the desired conclusion.

$(\Leftarrow)$ Let us observe that, since central elements are characterized by equations and equations are preserved by direct products, if $c_{1}$ and $c_{2}$ are central elements of two integral involutive near semirings $\mathbf{R}_{1}$ and $\mathbf{R}_{2}$, then $\left(c_{1}, c_{2}\right) \in \operatorname{Ce}\left(\mathbf{R}_{1} \times \mathbf{R}_{2}\right)$. Suppose $c \in \operatorname{Ce}\left(R_{e}\right)$, the image of $c$ under the isomorphism of Theorem 13 is $(c, 0)$. On the other hand, 0 is always central element, therefore we have that $(c, 0)$ is a central element in $\mathbf{R}_{e} \times \mathbf{R}_{e^{\prime}}$, implying that $c \in \operatorname{Ce}(\mathbf{R})$, as $\mathbf{R} \cong \mathbf{R}_{e} \times \mathbf{R}_{e^{\prime}}$. 
We have seen, in Proposition 3, that $\operatorname{Ce}(\mathbf{R})=\langle C e(R),+, \cdot, \alpha, 0,1\rangle$ is a Boolean algebra. Therefore it makes sense to consider the set of its atoms, which we denote by $\operatorname{At}(\mathbf{R})$.

LEMMA 8

If $\mathbf{R}$ is an involutive integral near semiring and $e \in \operatorname{At}(\mathbf{R})$, an atomic central element of $\mathbf{R}$, then $\operatorname{At}\left(\mathbf{R}_{\alpha(e)}\right)=\operatorname{At}(\mathbf{R}) \backslash\{e\}$.

Proof. (つ) Suppose that $e$ is an atom of the Boolean algebra $\mathrm{Ce}(\mathbf{R})$. Then, for any other atomic central element $c \in \mathbf{R}, c \wedge e=c \cdot e=e \cdot c=0$, therefore $\alpha(e)+\alpha(c)=1$. Furthermore, $c=1 \cdot c=(e+$ $\alpha(e)) \cdot c=(e \cdot c)+(\alpha(e) \cdot c)=0+(\alpha(e) \cdot c)=\alpha(e) \cdot c$, which shows that $c \leq \alpha(e)$. Thus, by Proposition $4, c \in \mathbf{R}_{\alpha(e)}$. We have to show that $c$ is also an atom. So, suppose $d$ is a central element of $\mathbf{R}_{\alpha(e)}$ such that $d<c$, then, by Proposition $4, d$ is a central element of $\mathbf{R}$ and as, by assumption, $c \in \operatorname{At}(\mathbf{R})$, then necessarily $d=0$, showing that $c$ is also an atom in $\mathbf{R}_{\alpha(e)}$.

( $\subseteq$ ) Suppose $c \in A t\left(\mathbf{R}_{\alpha(e)}\right)$, then in particular $c$ is a central element of $\mathbf{R}_{\alpha(e)}$ and, by Proposition 4, $c \in \mathrm{Ce}(\mathbf{R})$. Let $d \in \mathrm{Ce}(\mathbf{R})$, with $c<d$, then we have $d \leq \alpha(e)$ and therefore $d \in \operatorname{Ce}\left(\mathbf{R}_{\alpha(e)}\right)$ by Proposition 4. As, by assumption, $c \in \operatorname{At}\left(\mathbf{R}_{\alpha(e)}\right)$ then $d=0$, which shows that $c$ is an atomic central. We finally claim that $c \neq e$. Indeed, suppose by contradiction that $c=e$, then since $c \leq \alpha(e)$ we have $e \leq \alpha(e)$, i.e. $e=e \cdot \alpha(e)=0$ which is a contradiction, as $e$ is atomic central by hypothesis.

Lemma 8 will be useful in proving the following

THEOREM 16

Let $\mathbf{R}$ be an involutive integral near semiring such that $\mathrm{Ce}(\mathbf{R})$ is an atomic Boolean algebra with countably many atoms, then

$$
\mathbf{R}=\prod_{e \in A t(\mathbf{R})} \mathbf{R}_{e}
$$

is a decomposition of $\mathbf{R}$ as a product of directly indecomposable algebras.

Proof. The claim is proved by induction on the number of elements of $\operatorname{At}(\mathbf{R})$. If 1 is the only central atomic element, then $\mathbf{R}$ is directly indecomposable and clearly $\mathbf{R}=\mathbf{R}_{1}$. If there is an atomic central element $e \neq 1$, then $\mathbf{R}=\mathbf{R}_{e} \times \mathbf{R}_{\alpha(e)}$ by Theorem 13. On the other hand $\operatorname{Ce}\left(\mathbf{R}_{e}\right)=\{0, e\}$, because if $\mathbf{R}_{e}$ had another element, say $d$, then $d$ would be a central element of $\mathbf{R}$ in virtue of Proposition 4 and $0<d<e$ contradicting the fact that $e$ is an atom. Consequently $\mathbf{R}_{e}$ is directly indecomposable. By Lemma 8, $\operatorname{At}\left(\mathbf{R}_{\alpha(e)}\right)=A t(\mathbf{R}) \backslash\{e\}$ and by induction hypothesis, $\mathbf{R}_{\alpha(e)}=\prod_{c \in A t\left(\mathbf{R}_{\alpha(e)}\right)} \mathbf{R}_{c}$, whence our result follows.

\section{Acknowledgements}

S.B. acknowledges the Italian Ministry of Scientific Research (MIUR) for the support within the PRIN project Theory of Rationality: logical, epistemological and computational aspects. I.C. is supported by the bilateral Project 'New perspectives on residuated posets' financed by Austrian Science Fund (FWF), project I 1923-N25, and the Czech Science Foundation (GACR): project 1534697L. A.L. gratefully acknowledges the support of the Italian Ministry of Scientific Research (MIUR) within the FIRB project 'Structures and Dynamics of Knowledge and Cognition', Cagliari: F21J12000140001. Finally, we all thank Francesco Paoli for his valuable suggestions. 


\section{References}

[1] L. P. Belluce and A. Di Nola. Commutative rings whose ideals form an mv-algebra. Mathematical Logic Quarterly, 55, 468-486, 2009.

[2] L. P. Belluce, A. Di Nola and A. R Ferraioli. MV-semirings and their sheaf representations. Order, 30, 165-179, 2013.

[3] L. Beran. Orthomodular Lattices: Algebraic Approach. Mathematics and its Applications. Springer, 2011.

[4] S. Bonzio, I. Chajda and A. Ledda. Orthogonal relational systems. Soft Computing, 1-9, doi: 10.1007/s00500-015-1999-4.

[5] I. Chajda. Basic algebras, logics, trends and applications. Asian-European Journal of Mathematics, 8, 1550040-1550086, 2015.

[6] I. Chajda, G. Eigenthaler and H. Länger. Congruence Classes in Universal Algebra. Research and exposition in mathematics. Heldermann, 2003.

[7] I. Chajda, R. Halaš and J. Kühr. Semilattice Structures. Research and exposition in mathematics. Heldermann, 2007.

[8] I. Chajda and M. Kolař' $1 k$. Interval basic algebras. Novi Sad Journal of Mathematics, 39, 71-78, 2009.

[9] I. Chajda and H. Länger. Commutative basic algebras and coupled near semirings. Soft Computing, 19, 1129-1134, 2015.

[10] I. Chajda and H. Länger. A representation of basic algebras by coupled right near semirings. Acta Scientiarum Mathematicsrum (Szegel), to appear.

[11] A. Di Nola and B. Gerla. Algebras of Lukasiewicz's logic and their semiring reducts. Contemporary Mathematics, 377, 131-144, 2005.

[12] A. Di Nola and C. Russo. The semiring-theoretic approach to MV-algebras: a survey Fuzzy Sets and Systems, 281, 134-154, 2015.

[13] B. Gerla. Many valued logics and semirings. Neural Networks World, 467-480, 2003.

[14] I. Chajda, J. Gil-Fèrez, M Kolař' 'k, R. Giuntini R., A. Ledda and F. Paoli. On some properties of directoids. Soft Computing, 19, 955-964, 2015.

[15] G. Kalmbach. Orthomodular Lattices. L.M.S. monographs. Academic Press, 1983.

[16] A. Ledda, F. Paoli and A. Salibra. On semi-Boolean-like algebras. Acta Universitatics Palackianae Olomucensis, 52, 101-120, 2013.

[17] A. I. Mal'cev. On the general theory of algebraic systems. Mathematicheskii Sbornik, 35, 3-20, 1954.

[18] A. Salibra, A. Ledda, F. Paoli and T. Kowalski. Boolean-like algebras. Algebra Universalis, 69, 113-138, 2013.

[19] M. H. Stone. The theory of representation for boolean algebras. Transactions of the American Mathematical Society, 40, 37-111, 1936.

[20] D. Vaggione. Varieties in which the pierce stalks are directly indecomposable. Journal of Algebra, 184, 424-434, 1996.

Received 3 December 2015

\section{Appendix}

In Section 6, we mentioned that the axiomatization of central element for the variety of integral involutive near semirings can be reduced to a minimal set of two identities. Indeed, Theorem 14 


\section{Near semirings}

states that an element $e$ of an involutive near semiring is central if and only if it satisfies the following identities:

(1) $(e \cdot \alpha(x))+(\alpha(e) \cdot \alpha(y))=\alpha((e \cdot x)+(\alpha(e) \cdot y))$;

(1) $(e \cdot(x \cdot z))+(\alpha(e) \cdot(y \cdot u))=((e \cdot x)+(\alpha(e) \cdot y)) \cdot((e \cdot z)+(\alpha(e) \cdot u))$.

Here we provide a justification of the minimality of this axiomatization. In fact, we will show in this section that identities (1) and (2) are independent.

EXAMPLE 3

The integral involutive near semiring $\mathbf{A}$, whose sum, multiplication and the antitone involution $\alpha$ are defined in the following tables, satisfies (1) but not (2).

\begin{tabular}{|c|c|c|c|c|c|c|c|c|c|c|c|c|c|c|c|}
\hline$\alpha$ & & + & 0 & 1 & $e$ & $a$ & $b$ & $c$ & . & 0 & 1 & $e$ & $a$ & $b$ & $c$ \\
\hline 0 & 1 & 0 & 0 & 1 & $e$ & $a$ & $a$ & $c$ & 0 & 0 & 0 & 0 & 0 & 0 & 0 \\
\hline 1 & 0 & 1 & 1 & 1 & 1 & 1 & 1 & 1 & 1 & 0 & 1 & $e$ & $a$ & $b$ & $c$ \\
\hline$e$ & $a$ & $e$ & $e$ & 1 & $e$ & 1 & 1 & $e$ & $e$ & 0 & $e$ & $e$ & 0 & $c$ & $c$ \\
\hline$a$ & $e$ & $a$ & $a$ & 1 & 1 & $a$ & $a$ & $a$ & $a$ & 0 & $a$ & 0 & $a$ & $a$ & 0 \\
\hline$b$ & $c$ & $b$ & $a$ & 1 & 1 & $\mathrm{a}$ & $\mathrm{a}$ & $\mathrm{a}$ & $b$ & 0 & $b$ & 0 & $a$ & $a$ & 0 \\
\hline$c$ & $b$ & $c$ & $c$ & 1 & $e$ & $a$ & $a$ & $c$ & $c$ & 0 & $c$ & 0 & 0 & 0 & \\
\hline
\end{tabular}

It is routine to check that $\mathbf{A}$ is an integral involutive near semiring, satisfying also identity (1). A counterexample to identity (2) is given by setting: $x=b, z=1$ and $y=u=0$.

EXAMPLE 4

The integral involutive near semiring $\mathbf{B}$, whose sum, multiplication and the antitone involution $\alpha$ are defined in the following tables, satisfies (2) but not (1).

\begin{tabular}{l|l}
$\alpha$ & \\
\hline 0 & 1 \\
$a$ & $a$ \\
1 & 0
\end{tabular}

\begin{tabular}{c|ccc}
+ & 0 & 1 & $a$ \\
\hline 0 & 0 & 1 & $a$ \\
1 & 1 & 1 & 1 \\
$a$ & $a$ & 1 & $a$
\end{tabular}

\begin{tabular}{c|ccc}
$\cdot$ & 0 & 1 & $a$ \\
\hline 0 & 0 & 0 & $a$ \\
1 & 0 & 1 & $a$ \\
$a$ & 0 & $a$ & $a$
\end{tabular}

It is routine to check that $\mathbf{B}$ is an integral involutive near semiring satisfying Equation (2). A counterexample to identity (1) is given by setting $e=0$ and $x=y=z=a$.

As a consequence of Examples 3 and 4 we conclude that

Corollary 5

Equations (1) and (2) in Theorem 14 are independent. 